CORRECTION

\title{
Chronic lymphocytic leukaemia
}

Thomas J. Kipps, Freda K. Stevenson, Catherine J. Wu, Carlo M. Croce, Graham Packham,

William G. Wierda, Susan O'Brien, John Gribben and Kanti Rai

Nature Reviews Disease Primers 3, 16096 (2017)

In the version of the article originally published, a typographical error has now been corrected. The statement now reads: Approval of ibrutinib as initial therapy was based on the results of a randomized trial that showed a significant improvement in median PFS and overall survival in patients $\geq 65$ years of age without del(17p) who were treated indefinitely with ibrutinib than in patients treated for up to 48 weeks with chlorambuci ${ }^{146}$. 